\title{
CARCINOMA DEL CUELLO UTERINO ESTADO II
}

\section{Instituto Nacional de Cancerología}

\section{Doctor Germán Jordan Abondano *}

Con el fin de obtener una idea fundamental de los resultados logrados con el tratamiento que se sigue de manera notablemente uniforme en los casos de Ca de Cérvix Estado II en el período anterior al uso del Co 60, se revisaron la totalidad de' las historias que habían sido anotadas en las tarjetas de kárdex del Servicio de Ginecología hasta 1958, para intentar controles de más de 5 años. Las más antiguas fueron de 1944, antes de este año no había sido anotada ninguna. Estas historias suponen por lo menos que tuvieron un control después del tratamiento, las que no tuvieron control alguno, desde luego, no tenían por qué estar anotadas.

Se encontraron 76 casos, de los cuales se descartó 1 porque se comprobó en la revisión de las placas histológicas y a causa de no haber encontrado tumor en la pieza quirúrgica, que se trataba de un falso diagnóstico y no había tumor en las biopsias iniciales.
De estos 75 casos, 55 o sea el 73.33 por ciento fueron tratados con actinoterapia y 20 o sea el $26.67 \%$ fueron tratados inicialmente con cirugía, con operación de tipo radical (histerolinfadenectomía radical) en 19 casos e histerectomía total en 1 caso, con biopsia de los ganglios pélvicos. No está claro qué criterio se siguió para indicar uno u otro tratamiento, pero al parecer no se basó generalmente en diferencias clínicas sino en algunos casos en los cuales la obesidad, edad de las enfermas o enfermedades asociadas, contraindicaban el acto quirúrgico y en otros la dificultad de las enfermas para pagar alojamiento en la ciudad no permitía el tratamiento roentgenterapico ambulatorio.

Las técnicas quirúrgicas fueron realizadas por el grupo de planta del Servicio con un criterio unificado y un entrenamiento suficiente. Las técnicas de radioterapia fueron prácticamente iguales en todos los casos con aplicación de

* Ginecólogo del Instituto Nacional de Cancerología, Bogotá. Profesor Auxiliar de Ginecología y Obstetricia. Hospital de San Ignacio. Universidad Javeriana (Bogotá). 
rayos $\mathrm{X}$, roentgenterapia convencional con aparatos de 190 klv 10 ma y aparato de $240 \mathrm{klv}, 20 \mathrm{ma}$ en dosis calculadas entre 3.500 y 4.000 mgs-hr en el centro de la pelvis y tratamiento con radium por el método de Regaut con colpóstatos cargados en los dos corchos laterales con tubos de $13,33 \mathrm{mgr}-\mathrm{h}$ radium, complementados a veces con un corcho central en la aplicación continua y con una sonda intrauterina con 2 o 3 tubos de radium de las mismas especificaciones. La dosis total de radium precervical fluctuó entre 3.800 y 4.000 mgrs-hr y la dosis intracavitaria fue dada en las mismas dosis.

La conducta en radioterapia fue seguida por un grupo de radioterapeutas de planta con criterio unificado. En algunos casos, como se verá más adelante, la intervención quirúrgica fue complementada por roentgenterapia profunda cuando la linfadenectomía dio ganglios metastásicos y en el caso de la histerectomía total.

Tres (3) casos tratados por reproducción después de la intervención a los 5 meses, 21/2 años y 4 años evolucionan desfavorablemente dos de ellos $\mathrm{y}$ en 1 se obtiene un efecto paliativo de 2 años, al cabo de los cuales el tumor sigue en evolución.

EDAD:

$\begin{array}{rrrrr}20 & \text { a } & 29 & \text { años } & 5 \\ 30 & \text { a } & 39 & ” & 22 \\ 40 & \text { a } & 49 & , & 28 \\ 50 & \text { a } & 59 & ” & 15 \\ 60 & \text { a } & 69 & , & 4 \\ 70 & \text { a } & 79 & , & 1\end{array}$

Se observa que la tercera, cuarta y quinta décadas son las más afectadas por la incidencia de la lesión tumoral.

\section{Clasificación histopatológica:}

Ca escamocelular 68

Adenocarcinoma 2

Ca indiferenciado

Ca basocelular

4

1

Paridad:

$\begin{array}{lr}\text { Nulíparas } & 2 \\ \text { Sin dato } & 3 \\ 4 \text { o menos } & 18 \\ 5 \text { o más } & 42\end{array}$

Así pues la mayoría eran grandes multíparas y debe agregarse que 20 de ellas habían tenido 10 o más partos. 5 de estas enfermas recibieron tratamiento estando embarazadas. 1 de ellas tratada con cirugía sobrevive 11 años después, otra tratada con cirugía, radium y rayos $\mathrm{X}$ sobrevive con un último control a los 4 años y 6 meses, la tercera tratada con radium $y$ rayos $X$ se le practica una histerectomía un año después sin encontrar tumor residual, la cuarta, después de intervención, continúa en evolución y se declara intratable y en la quinta, después de cirugía, se reproduce a los 4 años; es tratada con radium y rayos $X$ y sobrevive 2 años más con $T$. en evolución. Puede en general decirse que los mejores resultados se obtienen en estos casos utilizando al máximo y en forma combinada el tratamiento quirúrgico y el radioterápico.

Casos tratados con cirugía

20 casos fueron inicialmente tratados con cirugía, 19 de ellos como ya se 
dijo, con histerolinfadenectomía radical y 1 con histerectomía total complementada con rayos $\mathrm{X}$ intravaginal en el postoperatorio.

\section{Controles}

11 casos tuvieron control de 5 años.

10 casos sobrevivían a los 5 años $\sin$ tumor y 1 con $T$. en evolución. De estos 10 casos, 3 fueron tratados con roentgenterapia postoperatoria, dos de ellos por ganglios positivos y 1 porque solamente se le había practicado histerectomía total. El caso reproducido a los $41 / 2$ años fue tratado con radium y rayos $X 11 \frac{1}{2}$ años despuís (es decir, a los $6 \frac{1}{2}$ años del tratamiento inicial); en su último control se declaró intratable.

9 casos no llegaron a un control de 5 años.

3 casos por muerte, todos con reproducción del $\mathrm{T}$.

2 casos sin control, no volvieron a consulta.

4 casos con controles de 6,18 y 30 meses con reproducción, no regresaron a más controles.

\section{Fístulas}

De las 20 enfermas 5 hicieron fístulas vésico-vaginales y una urétero-vaginal.

\section{Shock operatorio}

En 2 casos se presentó shock grave, uno de ellos con recuperación completa y el otro con recuperación parcial saliendo la enferma en muy malas condiciones 1 mes después y con muerte a los pocos días de salir del hospital.
RESUMEN DE LOS

CASOS QUIRURGICOS

\begin{tabular}{lrr}
\hline & $\%$ \\
\hline $\begin{array}{l}\text { Total de casos } \\
\text { Supervivencia de } 5 \text { años }\end{array}$ & 20 & 100 \\
$\quad$ sin T. & 10 & 50 \\
Supervivencia de 5 años & & \\
$\quad$ con T. & 1 & \\
Fístulas operatorias & 6 & 30 \\
\hline
\end{tabular}

Casos tratados con radioterapia

35 casos fueron tratados inicialmente con radioterapia, en 4 de ellos entre 6 meses y 1 año después, considerándolos blanqueados y en un estudio clínico quirúrgico se practica histerectomía posterior no encontrando en ninguno de los 4 casos $T$. residual.

\section{Controles}

47 casos tuvieron un control de 5 o más años.

46 casos con supervivencia de 5 años $\sin$ T., $83.63 \%$.

1 control de 5 años con $T$. en evolución.

8 no llegaron al control de 5 años. De estas 3 habían sido operadas meses después del tratamiento sin encontrar T. en la pieza quirúrgica y 2 de ellas fueron a su último control a los 41/2 años de tratamiento $\mathrm{y}$ se anotaron como casos sin que estuvieran en evolución; es decir casi con certeza se podrían agregar a los casos sin T. a los 5 años, pero no se agregan para atenerse a lo establecido en estas estadísticas.

12 casos de los 47 tienen 10 o más años de control sin reproducción y 9 
casos tienen 15 o más de control sin reproducción.

Debe agregarse que de estos casos uno se reproduce a los 10 años y se considera intratable, otro presenta una metástasis a vértebra lumbar 8 años más tarde y es tratada con rayos $\mathrm{X}$ sin volver a control. Una enferma hizo fístula rectal post-radioterapia.

En 1 caso 81/2 años después de tratado el Ca de cérvix y sin reproducción se encuentra un Ca de seno.

En 1 caso 16 años después del tartamiento y sin $\mathrm{T}$. cervical en evolución presenta un Ca de vulva.

\begin{tabular}{lcc}
\hline & $\%$ \\
\hline $\begin{array}{l}\text { Total de casos } \\
\text { Supervivencia de } 5\end{array}$ & 55 & 100 \\
$\quad$ años sin T. & 46 & 83.63 \\
Supervivencia de 5 & & \\
$\quad$ años con T. & 1 & \\
Fístula rectal & 1 & \\
\hline
\end{tabular}

\section{Resumen y conclusiones}

Se revisaron 76 casos de $\mathrm{Ca}$ de cérvix Estado II, de los cuales en total considerando los tratamientos quirúrgicos y roentgenterápicos, 56 casos sobrevivieron 5 o más años con un porcentaje de sobrevivencia a los 5 años sin tumor, de $74.66 \%$. 2 casos más sobrevivieron 5 años pero con $T$. en evolución.
De los 55 casos tratados con radium y rayos $X, 46$ sobrevivieron 5 o más años sin tumor, con un porcentaje de sobrevivencia a los 5 años del 83.63 por ciento,

De los 20 casos tratados quirúrgicamente, 10 casos sobrevivían a los 5 años con una sobrevivencia a los 5 años en un porcentaje del $50 \%$.

De los 20 casos quirúrgicos, 6, es decir el $30 \%$ presentaron fístulas urinarias postoperatorias.

Puede decirse fundamentado en los datos anteriores que el resultado del tratamiento del Ca de cérvix Estado II con roentgenterapia convencional profunda y radium con los equipos y técnicas utilizados en el Instituto Nacional de Cancerología de Colombia es excelente con una sobrevivencia a los 5 años, comparable a la publicada por otras entidades similares y con ventaja.

El tratamiento quirúrgico, a pesar de dar resultados buenos con una sobrev: vencia a los 5 años del $50 \%$, da un porcentaje notable de complicaciones fistulosas y es inferior a los anteriores. Debe agregarse que desde hace varios años se ha prescindido de la cirugía en estos casos y sólo se realiza en los Estados I y que se han depurado y mejorado las técnicas quirúrgicas, disminuyendo en ista forma la morbilidad operatoria. 